\title{
Sensitivity Analysis and Modeling the Impact of Screening on the Transmission Dynamics of Human Papilloma Virus (HPV)
}

\author{
Eshetu Dadi Gurmu, Purnachandra Rao Koya* \\ Department of Mathematics, Wollega University, Nekemte, Ethiopia \\ Email address: \\ eshetudadi1@gmail.com (E. D. Gurmu),drkpraophd@gmail.com (P. R. Koya) \\ ${ }^{*}$ Corresponding author
}

\section{To cite this article:}

Eshetu Dadi Gurmu, Purnachandra Rao Koya. Sensitivity Analysis and Modeling the Impact of Screening on the Transmission Dynamics of Human Papilloma Virus (HPV). American Journal of Applied Mathematics. Vol. 7, No. 3, 2019, pp. 70-79.

doi: 10.11648/j.ajam.20190703.11

Received: June 21, 2019; Accepted: July 22, 2019; Published: August 26, 2019

\begin{abstract}
In this paper, a mathematical model on the Human Papilloma Virus (HPV) governed by a system of ordinary differential equations is developed. The aim of this study is to investigate the role of screening as a control strategy in reducing the transmission of the disease. It is shown that a solution for the system of model equations exists and is unique. Further, it is shown that the solution is both bounded and positive. Hence, it is claimed that the model developed and presented in this paper is biologically meaningful and mathematically valid. The model is analyzed qualitatively for verifying the existence and stability of disease free and endemic equilibrium points using threshold parameter that governs the disease transmission. Furthermore, sensitivity analysis is performed on the key parameters driving Human Papilloma Virus and to determine their relative importance and potential impact on the dynamics of Human Papilloma Virus. Numerical result shows that Human Papilloma Virus infection is reduced using screening strategies. Due to the presence of interventions, the number of susceptible cells decreases implying that, most of the susceptible cells are screened. Similarly, the number of unaware infected cells decreases. This happens because unaware cells become aware after screening. The screened infected cells initially increase and then start to diminish after the equilibrium point. This is because many people from screened class recovered through treatment. Also, the number of cells with cancer decreases and this may be due to disease induced death. Furthermore, the number of recovered cells increases because there are two ways of recovering, through immune system or treatment. With $\mathfrak{R}_{0}=0.5677$, implies that screening can reduce the transmission of the disease in the population when $\mathfrak{R}_{0}<1$.
\end{abstract}

Keywords: HPV Infection, Sensitivity Analysis, Screening, Basic Reproduction Number, Stability Analysis, Jacobian Matrix, Numerical Simulation

\section{Introduction}

Biology of HPV: Human Papilloma Virus (HPV) is the name of a group of viruses that includes more than 100 different types and also more than 40 of these viruses are the most common and sexually transmit in the world. Among them, HPV types 16, 18, 31 and 45 are referred to as "highrisk" and causes approximately $85 \%$ of cervical cancers. The targets of initial infection are the basal epithelial cells in the cervix. Subsequent binding and entry, viral material migrates to the nucleus and establishes the HPV genomes as multiplecopy extra chromosomal plasmids, which are maintained at approximately 20 to 100 copies per infected cell. Infected cells express viral proteins, E6 and E7, which interfere with the normal cell cycle, promoting proliferation and deactivating the tumor suppressor proteins p53 and pRb. Subsequent viral genome replication and cell division, one of the daughter cells migrates away from the basal layer and starts a program of differentiation. Unlike normal cells, HPVinfected cells undergo differentiations but remain active in the cell cycle. Some HPV infections of healthy hosts can be cleared by the immune system. However, many infections, especially by high risk strains such as HPV 16 and 18, become chronic. These persistent infections constantly shed 
HPV virions. Ultimately, the persistence of actively proliferating cells leads to the development of precancerous cells, which are at risk, in turn, of becoming cancer cells. With proper treatment, precancerous cells may be prevented from progressing to the cancerous stage [1].

Eradication HPV: Most of the HPV infections are asymptomatic and can feed away without treatment over the course of a few years. For instance, about $70 \%$ of HPV infections fed away within a year and $90 \%$ within two years. However, in some people infection can persist for many years and can cause warts or low risk genotype of HPV, while other types lead to different kinds of cancers or high risk genotype of HPV including cervical cancer [2-3]. AlthoughHPV itself cannot be treated, the cellular changes that come from any HPV infection canbe treated. For examples, genital warts, cervical, anal, and genital cancers can be treatedif the infection is diagnosed during the early stage of development. Pre-cancerous cell changes caused by HPV can be detected by Pap tests and treat individuals who are found already infected. The purpose of screening is to provide treatment for those who are found to have HPV infection [4].

Statistics of HPV: According to the National Cancer Registry cancer kills more people than HIV/AIDS, malaria and tuberculosis combined [5]. Statistics show that 18.1 million new cases, 9.6 million cancer related deaths, and 43.8 million people living with cancer in 2018. The number of new cases is expected to rise from 18 million to 22 million by 2030 and the number of global cancer deaths is projected to increase by $45 \%$ in the period from 2007 to 2030 [6]. Almost $80 \%$ of cervical cancer cases and deaths occur in poor countries. In Sub-Saharan Africa, cervical cancer accounts for $22.5 \%$ of all cancer cases in women, and the majority of women who develop cervical cancer live in rural areas [7]. Eastern Africa is one of the most heavily affected areas with an incidence of more than 30 cases per 100,000 women per year [8]. In Ethiopia, cancer accounts for about $5.8 \%$ of total national mortality. Although population-based data does not exist in the country except for Addis Ababa, it is estimated that the annual incidence of cancer is around 60,960 cases and the annual mortality over 44,000 . The most prevalent cancers in Ethiopia among the entire adult population are breast cancer $(30.2 \%)$, cancer of the cervix $(13.4 \%)$ and colorectal cancer $(5.7 \%)$. About two-thirds of annual cancer deaths occur among women [9].

Importance of Modeling: A mathematical model is a description of a system using mathematical tools and language. Mathematical models are of great importance in the natural sciences, including biology and epidemiology. They help us to gain new understanding about a system, organize and make sense of biological data, obtain the response behavior of the system, seek optimal performance and intervention strategies, and make predictions about the system. Mathematical modeling of infectious diseases began in 1760s with Daniel Bernoulli's modeling of smallpox. Since then, mathematical models have been developed to simulate the spread of a wide range of infectious diseases, such as
HIV, tuberculosis, malaria and influenza to name but a few examples. These mathematical models have been developed to address a range of questions that cannot be answered through the use of traditional epidemiological methods.

Survey of Modeling: Many mathematical models have been developed to analyze the dynamics of transmission of HPV infection and its associated health problems, and as well study the impact of some control strategies against the virus. It is an essential and effective way to totally understand the real-world problems by establishing mathematical models and analyzing their dynamical behaviors. Old and recent studies such as [10] developed a mathematical model to investigate the impact of vaccination against human Papilloma virus, accounting for a wide-spread childhood vaccination program that may be supplemented by voluntary adult vaccination. Also, [11] formulated an SIS model for human Papilloma virus transmission with vaccination as a control strategy and [12] developed a dynamic model for the heterosexual transmission of Human Papillomavirus types 16 and 18 , which are covered by available vaccines. Moreover, Kermack and McKendrick [13] develop SIR cancer model and some other recent studies by Akram et al [14] develop the mathematical model that describe interaction between uninfected tumor cells and infected tumor cell and modified with count treatment of cells by chemotherapy and recovery class [15].

Motivation for the present Model: However, none of them considered the mathematical model for analyzing screening on reducing HPV infection in the population. In this study a mathematical model will be developed to determine the effectiveness of screening in attempting to reduce HPV transmission. The purpose of screening is to provide treatment for those who are found to have HPV infection. So in this study the model in [15] is modified by adding unaware infected and screening infected class instead of treatment and cervical cancer class.

\section{Model Formulation}

Mathematical modeling process requires translation of a biological scenario into a mathematical problem. It begins with a clear description of the processes based on the scientists understanding of the system. The translation into mathematical equations should be made with a specific goal or biological question in mind. Then the verbal description of the system is encoded in mathematical equations. Mathematical models usually consist of parameters and variables that are connected by relationships. Variables are abstractions of the system properties that can be quantified or measured and parameters describe the rate of variables.

The model of Human Papilloma Virus (HPV) infection and cervical cancer development in the cervix consists of five class: ( $i)$ Susceptible class denoted by $S(t)$ consists of cells which are capable of becoming infected (ii) Unaware infected class denoted by $I_{u}(t)$ consists of cells which are unaware infected with virus and are also infectious (iii) Screened infected class denoted by $I_{S}(t)$ consists of cells 
which are screened infected with virus and provide treatment for those who are found to have HPV infection (iv) Recovered class denoted by $R(t)$ consists of recovered cellsand $(v)$ Cervical Cancer class denoted by $C(t)$ consists of cervical cancer cells.

Here, a mathematical model of the Human Papilloma Virus is constructed based on the following assumptions:

(i) Susceptible individuals are recruited into the population at a constant rate $\Pi$.

(ii) Susceptible cells may acquire HPV infection at rate $\lambda$ when they come into effective contact with infectious cells at the rate $\beta$ that may lead to infection. The force of infection in the model is given as $\lambda=\beta\left[I_{u}(t)+I_{s}(t)\right] / N$.

(iii) The unaware infected cells are screened and join the screened infected class at a rate $\alpha$.

(iv) Some of the unaware infected cells progress to cervical cancer at a rate $\epsilon$ and others recover naturally through body immune system at a rate $\delta$.

(v) The screened infected cells are treated at a rate $\omega$ and move to recovery class or may progress to develop cervical cancer as a result of failure of the treatment used at a rate $\phi$ thus moving to cervical cancer class.

(vi) Recovered cells revert to the susceptible class after losing their immunity at a rate $\varphi$.

(vii) Cervical cancer cells die of infection at a rate $\gamma$.

(viii) All types of cells suffer natural mortality at a rate $\mu$.

(ix) All parameters in the model are positive.

Table 1. Notations and description of model variables.

\begin{tabular}{ll}
\hline Variable & Description \\
\hline $\mathrm{S}(\mathrm{t})$ & Population size of susceptible cells \\
$\mathrm{I}_{\mathrm{u}}(\mathrm{t})$ & Population size of unaware infected and infectious cells \\
$\mathrm{I}_{\mathrm{s}}(\mathrm{t})$ & Population size of screened infected cells \\
$\mathrm{R}(\mathrm{t})$ & Population size of recovered cells \\
$\mathrm{C}(\mathrm{t})$ & Population size of cervical cancer cells \\
\hline
\end{tabular}

Table 2. Notations and description of model parameters.

\begin{tabular}{|c|c|}
\hline Parameter & Description \\
\hline$\Pi$ & $\begin{array}{l}\text { Recruited rate of susceptible cells. With this rate new cells } \\
\text { will born and they will enter into susceptible class }\end{array}$ \\
\hline$\beta$ & $\begin{array}{l}\text { Transmission rate of infection. With this rate cells transfer } \\
\text { from compartment } S \text { to } I\end{array}$ \\
\hline$\lambda$ & Infection rate or force of infection \\
\hline$\alpha$ & With this rate cells transfer from compartment $I_{u}$ to $I_{s}$ \\
\hline$\omega$ & $\begin{array}{l}\text { Treatment rate. With this rate cells transfer from } \\
\text { compartment } I_{s} \text { to } R\end{array}$ \\
\hline$\varphi$ & $\begin{array}{l}\text { Recovery rate. With this rate cells transfer from } \\
\text { compartment } R \text { to } S\end{array}$ \\
\hline$\epsilon$ & With this rate cells transfer from compartment $I_{u}$ to $C$ \\
\hline$\phi$ & With this rate cells transfer from compartment $I_{s}$ to $C$ \\
\hline$\gamma$ & $\begin{array}{l}\text { Death rate due to infection. With this rate cells } \\
\text { of } C \text { compartment die of the disease. }\end{array}$ \\
\hline$\mu$ & $\begin{array}{l}\text { Natural death rate. With this rate cells of all the } \\
\text { compartments die naturally. }\end{array}$ \\
\hline
\end{tabular}

Upon including the basic assumptions together with the description of both model variables and parameters the schematic diagram of the modified model can be given as in Figure 1.

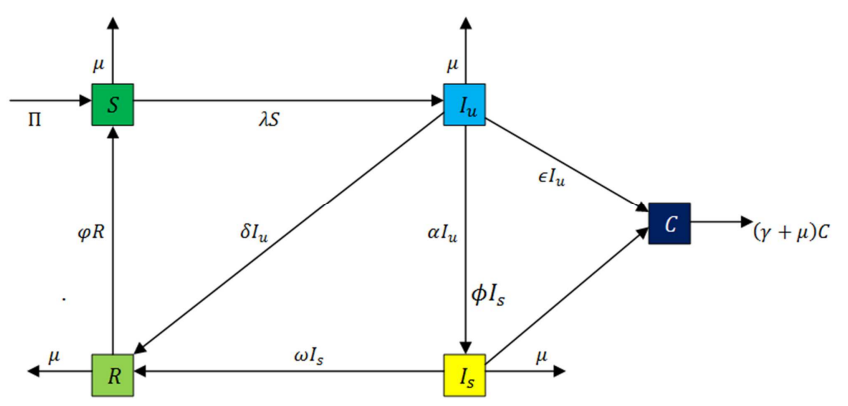

Figure 1. Schematic diagram of the model.

Based on the model assumptions, the notations of variables and parameters and the schematic diagram, the model equations are formulated and given as follows:

$$
\begin{gathered}
d S / d t=\Pi-(\lambda+\mu) S+\varphi R \\
d I_{u} / d t=\lambda S-(\epsilon+\alpha+\delta+\mu) I_{u} \\
d I_{s} / d t=\alpha I_{u}-(\omega+\phi+\mu) I_{s} \\
d C / d t=\epsilon I_{u}+\phi I_{s}-(\gamma+\mu) C \\
d R / d t=\delta I_{u}+\omega I_{s}-(\varphi+\mu) R
\end{gathered}
$$

The non-negative initial conditions of the system of model equations (1) - (5) are denoted by $S(0)>0, I_{u}(0) \geq$ $0, I_{S}(0) \geq 0, C(0) \geq 0, R(0) \geq 0$. This system consists of five first order non-linear ordinary differential equations.

\section{Mathematical Analysis of the Model}

The mathematical analysis of the model described by the system (1) - (5) is presented here. The model represented by the systems of differential equations $(1)-(5)$ will be analyzed in the feasible region and since the model represents the populations all the state variables and the parameters are assumed to be positive. The invariant region for the model (1) $-(5)$ is

$$
\Omega=\left\{\left(S, \quad I_{u}, \quad I_{s}, C, \quad R\right) \in \mathbb{R}_{+}^{5} ; \mathrm{N} \leq(\pi / \mu)\right\}
$$

Therefore, the solutions of the system of ordinary differential equations (1) - (5) are feasible for all $t>0$ if they enter the invariant region $\Omega$.

\subsection{Boundedness, Existence and Positivity of Solution}

In order to show that the model is biologically valid, it is required to prove that the solutions of the system of differential equations (1) - (5) are both positive and bounded for all time. It is done starting with Lemma 1.

Lemma 1 (Boundedness): Thesolutions of the system of model equations (1) - (5) are bounded. That is, the model variables $S(t), I_{u}(t), I_{s}(t), C(t)$, and $R(t)$ are bounded for all $t$.

Proof: Recall that each population size is bounded if and only if the total population size is bounded. Hence, in the present case it is sufficient to prove that the total population size $N=S(t)+I_{u}(t)+I_{s}(t)+C(t)+R(t)$ is bounded for all $t$. It can be begun by showing that all feasible solutions 
are uniformly bounded in a proper subset $\Omega \in \mathbb{R}_{+}^{5}$ where the feasible region $\Omega$ is given by $\Omega=\left\{\begin{array}{lll}S, & I_{u}, & I_{S}\end{array}, C, R\right) \in$ $\left.\mathbb{R}_{+}^{5} ; \mathrm{N} \leq(\Pi / \mu)\right\}$.

Now, summation of all the four equations (1) - (5) of the model givesdN $(\mathrm{t}) / \mathrm{dt}=\Pi-\mu \mathrm{N}(\mathrm{t})-\gamma \mathrm{C}$. It can be expressed without loss of generality, after eliminating the negative term $(-\gamma \mathrm{C})$ which is appearing on the right hand side, as an inequality as $\mathrm{dN}(\mathrm{t}) / \mathrm{dt} \leq[\Pi-\mu \mathrm{N}(\mathrm{t})]$. Equivalently this inequality can be expressed as a linear ordinary differential inequality as $\mathrm{dN}(\mathrm{t}) / \mathrm{dt}+\mu \mathrm{N}(\mathrm{t}) \leq \Pi$ giving general solution upon solving as $N(\mathrm{t}) \leq(\Pi / \mu)+c e^{-\mu t}$. But, the term $N(0)$ denotes the initial values of the respective variable i.e., $\mathrm{N}(\mathrm{t})=\mathrm{N}(0)$ at $\mathrm{t}=0$. Thus, the particular solution can be expressed as $\mathrm{N}(\mathrm{t}) \leq(\Pi / \mu)+[\mathrm{N}(0)-(\Pi / \mu)] e^{-\mu t}$. Further, it can be observed that $N(t) \rightarrow(\Pi / \mu)$ as $t \rightarrow \infty$. That is, the total population $\operatorname{size} N(t)$ takes off from the value $\mathrm{N}(0)$ at the initial time $t=0$ and ends up with the bounded value $(\Pi / \mu)$ as the time $t$ grows to infinity. Thus it can be concluded that $N(t)$ is bounded as $0 \leq N(t) \leq(\Pi / \mu)$.

Therefore, $(\Pi / \mu)$ is an upper bound of $N(t)$. Hence, feasible solution of the system of model equations (1) - (5) remains in the region $\Omega$ which is positively invariant set. Thus, the system is biologically meaningful and mathematically well posed in the domain $\Omega$. Further, it is sufficient to consider the dynamics of the populations represented by the model system (1) - (5) in that domain.

Therefore, it can be summarized the result of Lemma 1 as "the model variables $S(t), I_{u}(t), I_{S}(t), C(t)$, and $R(t)$ are bounded for all $t . "$

Lemma 2 (Existence) Solutions of the model equations (1) - (5) together with the initial conditions $S(0)>0, I_{u}(0) \geq$ $0, I_{S}(0) \geq 0, C(0) \geq 0, R(0) \geq 0$ exist in $\mathbb{R}_{+}^{5}$ i.e., the model variables $S(t), I_{u}(t), I_{s}(t), C(t)$, and $R(t)$ exist for all $t$ and will remain in $\mathbb{R}_{+}^{5}$.

Proof The right hand sides of the system of equations(1) (5) can be expressed as follows:

$$
\begin{aligned}
& f_{1}\left(S, \quad I_{u}, \quad I_{s}, C, \quad R\right)=\Pi-(\lambda+\mu) S+\varphi R \\
& f_{2}\left(S, \quad I_{u}, \quad I_{s}, C, \quad R\right)=\lambda S-(\epsilon+\alpha+\delta+\mu) I_{u} \\
& f_{3}\left(S, \quad I_{u}, \quad I_{s}, C, \quad R\right)=\alpha I_{u}-(\omega+\phi+\mu) I_{s} \\
& f_{4}\left(S, \quad I_{u}, \quad I_{s}, C, \quad R\right)=\epsilon I_{u}+\phi I_{s}-(\gamma+\mu) C \\
& f_{5}\left(S, \quad I_{u}, \quad I_{s}, C, \quad R\right)=\delta I_{u}+\omega I_{s}-(\varphi+\mu) R
\end{aligned}
$$

According to Derrick and Groosman theorem, let $\Omega$ denote the region $\Omega=\left\{\left(S, \quad I_{u}, I_{s}, C, R\right) \in \mathbb{R}_{+}^{5} ; \mathrm{N} \leq(\Pi / \mu)\right\}$. Then equations (1) - (5) have a unique solution if $\left(\partial f_{i}\right) /\left(\partial x_{j}\right), i, j=1,2,3,4,5$ are continuous and bounded in $\Omega$. Here using the notations $x_{1}=S, x_{2}=I_{u}, x_{3}=I_{s}, x_{4}=$ $C, x_{5}=R$ continuity and the boundedness is verified here under.

For $f_{1}$ :

$$
\begin{gathered}
\left|\left(\partial f_{1}\right) /(\partial S)\right|=\left|-\left[\beta\left(I_{u}+I_{S}\right) / N+\mu\right]\right|<\infty \\
\left|\left(\partial f_{1}\right) /\left(\partial I_{u}\right)\right|=|-(\beta S / N)|<\infty \\
\left|\left(\partial f_{1}\right) /\left(\partial I_{S}\right)\right|=|-(\beta S / N)|<\infty
\end{gathered}
$$

$$
\begin{gathered}
\left|\left(\partial f_{1}\right) /(\partial C)\right|=0<\infty \\
\left|\left(\partial f_{1}\right) /(\partial R)\right|=|\varphi|<\infty .
\end{gathered}
$$

For $f_{2}$ :

$$
\begin{gathered}
\left|\left(\partial f_{2}\right) /(\partial S)\right|=\left|\beta\left(I_{u}+I_{s}\right) / N\right|<\infty \\
\left|\left(\partial f_{2}\right) /\left(\partial I_{u}\right)\right|=|-(\epsilon+\alpha+\delta+\mu)|<\infty \\
\left|\left(\partial f_{2}\right) /\left(\partial I_{s}\right)\right|=\left|\beta\left(I_{u}+I_{s}\right) / N\right|<\infty \\
\left|\left(\partial f_{2}\right) /(\partial C)\right|=0<\infty \\
\left|\left(\partial f_{2}\right) /(\partial R)\right|=0<\infty .
\end{gathered}
$$

For $f_{3}$ :

$$
\begin{gathered}
\left|\left(\partial f_{3}\right) /(\partial S)\right|=0<\infty \\
\left|\left(\partial f_{3}\right) /\left(\partial I_{u}\right)\right|=|\alpha|<\infty \\
\left|\left(\partial f_{3}\right) /\left(\partial I_{s}\right)\right|=|-(\omega+\phi+\mu)|<\infty \\
\left|\left(\partial f_{3}\right) /(\partial C)\right|=0<\infty \\
\left|\left(\partial f_{3}\right) /(\partial R)\right|=0<\infty .
\end{gathered}
$$

For $f_{4}$ :

$$
\begin{gathered}
\left|\left(\partial f_{4}\right) /(\partial S)\right|=0<\infty \\
\left|\left(\partial f_{4}\right) /\left(\partial I_{u}\right)\right|=|\epsilon|<\infty \\
\left|\left(\partial f_{4}\right) /\left(\partial I_{s}\right)\right|=|\phi|<\infty \\
\left|\left(\partial f_{4}\right) /(\partial C)\right|=|-(\gamma+\mu)|<\infty \\
\left|\left(\partial f_{4}\right) /(\partial R)\right|=0<\infty .
\end{gathered}
$$

For $f_{5}$ :

$$
\begin{gathered}
\left|\left(\partial f_{5}\right) /(\partial S)\right|=0<\infty \\
\left|\left(\partial f_{5}\right) /\left(\partial I_{u}\right)\right|=|\delta|<\infty \\
\left|\left(\partial f_{5}\right) /\left(\partial I_{s}\right)\right|=|\omega|<\infty \\
\left|\left(\partial f_{5}\right) /(\partial C)\right|=0<\infty \\
\left|\left(\partial f_{5}\right) /(\partial R)\right|=|-(\gamma+\mu)|<\infty .
\end{gathered}
$$

Thus, all the partial derivatives $\left(\partial f_{i}\right) /\left(\partial x_{j}\right), i, j=$ $1,2,3,4,5$ exist, continuous and bounded in $\Omega$. Hence, by Derrick and Groosman theorem, a solution for the model (1) - (5) exists and is unique.

Lemma 3 (Positivity) Solutions of the model equations (1) - (5) together with the initial conditions $S(0)>0, I_{u}(0) \geq$ $0, I_{S}(0) \geq 0, C(0) \geq 0, R(0) \geq 0$ are always positive $(\mathrm{OR})$ the model variables $S(t), I_{u}(t), I_{s}(t), C(t)$, and $R(t)$ are positive for all $t$ and will remain in $\mathbb{R}_{+}^{5}$.

Proof Positivity is verified separately for each of the model variables $S(t), I_{u}(t), I_{s}(t), C(t)$, and $R(t)$.

Positivity of $S(t)$ : The model equation (1) given by $d S / d t=\Pi-\left[\beta\left(I_{u}+I_{S}\right) / N+\mu\right] S+\varphi R$ can be expressed without loss of generality, after eliminating the positive 
terms $(\Pi+\varphi R)$ which are appearing on the right hand side, as an inequality as $d S / d t \geq-\left[\beta\left(I_{u}+I_{s}\right) / N+\mu\right] S$. Using variables separable method and on applying integration, the solution of the foregoing differentially inequality can be obtained as $S(t) \geq e^{-\mu t-(\beta / N) \int\left(I_{u}+I_{S}\right) d t}$. Recall that an exponential function is always non-negative irrespective of the sign of the exponent, i.e., the exponential function $e^{-\mu t-(\beta / N) \int\left(I_{u}+I_{S}\right) d t}$ is a non-negative quantity. Hence, it can be concluded that $S(t) \geq 0$.

Positivity of $I_{u}(t)$ : The model equation (2) given by $d I_{u} / d t=\lambda S-(\epsilon+\alpha+\delta+\mu) I_{u}$ can be expressed without loss of generality, after eliminating the positive term $\left[\beta\left(I_{u}+\right.\right.$ $\left.I_{S}\right) S$ ] which are appearing on the right hand side, as an inequality as $d I_{u} / d t \geq-(\epsilon+\alpha+\delta+\mu) I_{u}$. Using variables separable method and on applying integration, the solution of the foregoing differentially inequality can be obtained as $I_{u}(t) \geq e^{-(\epsilon+\alpha+\delta+\mu) t}$. Recall that an exponential function is always non-negative irrespective of the sign of the exponent, i.e., the exponential function $e^{-(\epsilon+\alpha+\delta+\mu) t}$ is a non-negative quantity. Hence, it can be concluded that $I_{u}(t) \geq 0$.

Positivity of $I_{s}(t)$ : The model equation (3) given by $d I_{s} / d t=\alpha I_{u}-(\omega+\phi+\mu) I_{s}$ can be expressed without loss of generality, after eliminating the positive term $\left(\alpha I_{u}\right)$ which are appearing on the right hand side, as an inequality as $d I_{s} / d t \geq-(\omega+\phi+\mu) I_{s}$. Using variables separable method and on applying integration, the solution of the foregoing differentially inequality can be obtained as $I_{s}(t) \geq$ $e^{-(\omega+\phi+\mu) t}$. Recall that an exponential function is always non-negative irrespective of the sign of the exponent, i.e., the exponential function $e^{-(\omega+\phi+\mu) t}$ is a non-negative quantity. Hence, it can be concluded that $I_{s}(t) \geq 0$.

Positivity of $C(t)$ : The model equation (4) given by $d C / d t=\epsilon I_{u}+\phi I_{s}-(\gamma+\mu) C$ can be expressed without loss of generality, after eliminating the positive term $\left(\epsilon I_{u}+\right.$ $\left.\phi I_{s}\right)$ which are appearing on the right hand side, as an inequality as $d C / d t \geq-(\gamma+\mu) C$. Using variables separable method and on applying integration, the solution of the foregoing differentially inequality can be obtained as $C(t) \geq$ $e^{-(\gamma+\mu) t}$. Recall that an exponential function is always nonnegative irrespective of the sign of the exponent, i.e., the exponential function $e^{-(\gamma+\mu) t}$ is a non-negative quantity. Hence, it can be concluded that $C(t) \geq 0$.

Positivity of $R(t)$ : The model equation (5) given by $d R / d t=\delta I_{u}+\omega I_{s}-(\varphi+\mu) R$ can be expressed without loss of generality, after eliminating the positive term $\left(\delta I_{u}+\omega I_{s}\right)$ which are appearing on the right hand side, as an inequality as $d R / d t \geq-(\varphi+\mu) R$. Using variables separable method and on applying integration, the solution of the foregoing differentially inequality can be obtained as $R(t) \geq e^{-(\varphi+\mu) t}$. Recall that an exponential function is always non-negative irrespective of the sign of the exponent, i.e., the exponential function $e^{-(\varphi+\mu) t}$ is a nonnegative quantity. Hence, it can be concluded that $R(t) \geq$ 0 .

Thus, the model variables $S(t), I_{u}(t), I_{s}(t), C(t)$, and $R(t)$ representing population sizes of various types of cells are positive quantities and will remain in $\mathbb{R}_{+}^{5}$ for all $t$.

\subsection{The Disease Free Equilibrium (DFE)}

Disease free equilibrium points are steady state solutions where there is no disease in the population. Absence of disease implies that $I_{u}(t)=I_{s}(t)=C(t)=R(t)=0$ and the equilibrium pointsrequire that the right hand sides of the model equationsset equal to zero. These requirements reflect in reducing the model equations (1) $-(5)$ as $\Pi-(\lambda+\mu) S=0$ giving $S^{0}=\Pi /(\lambda+\mu)=(\pi / \mu)$ where $\lambda=\beta\left(I_{u}+I_{s}\right) / N=$ $\beta(0+0) / N=0$.

Thus, the disease-free equilibrium point of the model equation in (1) - (5) above is given by

$$
E_{0}=\left\{S^{0}, I_{u}{ }^{0}, I_{s}{ }^{0}, T^{0}, R^{0}\right\}=\{(\pi / \mu), 0,0,0,0\}
$$

\subsection{The Basic Reproduction Number $\left(\mathfrak{R}_{0}\right)$}

The basic reproduction number is denoted by $\mathfrak{R}_{0}$ and is defined as the expected number of people getting secondary infection among the whole susceptible population. It is computed using the next-generation matrix defined as in [15]. In this method $\Re_{0}$ is defined as the largest eigenvalue of the next generation matrix. Using the notation as in [15] for the model system $(1)-(5)$ the associated matrices $F$ and $V$ for the new infectious terms and the remaining transition terms are respectively given by:

$$
F_{i}=\left[\begin{array}{c}
{\left[\beta\left(I_{u}+I_{s}\right) S\right] / N} \\
0 \\
0
\end{array}\right] \text { and } V_{i}=\left[\begin{array}{c}
(\epsilon+\alpha+\delta+\mu) I_{u} \\
-\alpha I_{u}+(\omega+\phi+\mu) I_{s} \\
-\epsilon I_{u}-\phi I_{s}+(\gamma+\mu) C
\end{array}\right]
$$

The Jacobian matrices of $F_{i}$ and $V_{i}$ at the disease free equilibrium point $E_{0}$ take the form respectively as

$$
F=\left[\begin{array}{ccc}
\beta & \beta & 0 \\
0 & 0 & 0 \\
0 & 0 & 0
\end{array}\right] \text { and } V=\left[\begin{array}{ccc}
a & 0 & 0 \\
-\alpha & b & 0 \\
-\epsilon & -\phi & c
\end{array}\right]
$$

It can be verified that the matrix $V$ is non-singular as its determinant $\operatorname{det}[V]=a b c$ is non-zero and after some algebraic computations its inverse matrix is constructed as

$$
V^{-1}=\left[\begin{array}{ccc}
(1 / a) & 0 & 0 \\
(\alpha / a b) & (1 / b) & 0 \\
{[(\alpha \phi+\epsilon b) / \mu a]} & (\phi / b c) & (1 / c)
\end{array}\right]
$$

The product of the matrices $F$ and $V^{-1}$ can be computed as

$$
\begin{array}{r}
F V^{-1}=\left[\begin{array}{ccc}
\beta & \beta & 0 \\
0 & 0 & 0 \\
0 & 0 & 0
\end{array}\right]\left[\begin{array}{ccc}
(1 / a) & 0 & 0 \\
(\alpha / a b) & (1 / b) & 0 \\
{[(\alpha \phi+\epsilon b) / \mu a]} & (\phi / b c) & (1 / c)
\end{array}\right] \\
=\left[\begin{array}{ccc}
{[(\beta / a)+(\beta \alpha / a b)]} & (\beta / b) & 0 \\
0 & 0 & 0 \\
0 & 0 & 0
\end{array}\right]
\end{array}
$$

Now it is possible to calculate the eigenvalue to determine the basic reproduction number $\mathfrak{R}_{0}$ by taking the spectral radius of the matrix $F V^{-1}$. Thus, the eigenvalues are computed by evaluating $\operatorname{det}\left[F V^{-1}-\lambda I\right]=0$ or equivalently solving 


$$
\left|\begin{array}{ccc}
{[(\beta / a)+(\beta \alpha / a b)]-\lambda} & (\beta / b) & 0 \\
0 & -\lambda & 0 \\
0 & 0 & -\lambda
\end{array}\right|=0
$$

It reduces to the cubic equation for $\lambda$ as $\lambda^{2}[[(\beta / a)+$ $(\beta \alpha / a b)]-\lambda]=0$ giving the three eigenvalues as $\lambda_{1}=$ $[(\beta / a)+(\beta \alpha / a b)], \lambda_{2}=0, \lambda_{3}=0$. However, the largest eigenvalue here is $\lambda_{1}=[(\beta / a)+(\beta \alpha / a b)]$ and is the spectral radius as the threshold value or the basic reproductive number.

Thus, it can be concluded that the reproduction number of the model is $\Re_{0}=[\beta(b+\alpha) / a b]$.

$$
J=\left[\begin{array}{c}
-\left[\left[\beta\left(I_{u}+I_{s}\right) / N\right]+\mu\right] \\
{\left[\beta\left(I_{u}+I_{s}\right) / N\right]} \\
0 \\
0 \\
0
\end{array}\right.
$$

Therefore, the Jacobian matrix $J$ of model at the disease free equilibrium $E_{0}$ reduces to

$$
J\left(E_{0}\right)=\left[\begin{array}{ccccc}
-\mu & -\beta & -\beta & 0 & \varphi \\
0 & (\beta-a) & \beta & 0 & 0 \\
0 & \alpha & -b & 0 & 0 \\
0 & \epsilon & \phi & -c & 0 \\
0 & \delta & \omega & 0 & -d
\end{array}\right]
$$

Now, the eigenvalues of $J\left(E_{0}\right)$ are required to be found. The characteristic equation $\operatorname{det}\left[J\left(E_{0}\right)-\psi I\right]=0$ is expanded and simplified as follows:

$$
\begin{aligned}
& \left|\begin{array}{ccccc}
-\mu-\psi & -\beta & -\beta & 0 & \varphi \\
0 & (\beta-a)-\psi & \beta & 0 & 0 \\
0 & \alpha & -b-\psi & 0 & 0 \\
0 & \epsilon & \phi & -c-\psi & 0 \\
0 & \delta & \omega & 0 & -d-\psi
\end{array}\right|=0 \\
& -(\mu+\psi)\left|\begin{array}{cccc}
(\beta-a)-\psi & \beta & 0 & 0 \\
\alpha & -b-\psi & 0 & 0 \\
\epsilon & \phi & -c-\psi & 0 \\
\delta & \omega & 0 & -d-\psi
\end{array}\right|=0 \\
& (\mu+\psi)(d+\psi)\left|\begin{array}{ccc}
(\beta-a)-\psi & \beta & 0 \\
\alpha & -b-\psi & 0 \\
\epsilon & \phi & -c-\psi
\end{array}\right|=0 \\
& (\mu+\psi)(d+\psi)(-c-\psi)\left|\begin{array}{cc}
(\beta-a)-\psi & \beta \\
\alpha & -b-\psi
\end{array}\right|= \\
& (\mu+\psi)(d+\psi)(-c-\psi)[(\beta-a-\lambda)(-b-\lambda)-\alpha \beta]=0 \\
& \begin{array}{c}
(\mu+\psi)(d+\psi)(-c-\psi)\left[\psi^{2}+(a+b-\beta) \psi+a b(1\right. \\
\left.\left.-\Re_{0}\right)\right]=0
\end{array} \\
& (\mu+\psi)=0,(d+\psi)=0,(-c-\psi) \\
& =0, \psi^{2}+(a+b-\beta) \psi+a b\left(1-\Re_{0}\right) \\
& =0
\end{aligned}
$$

\subsection{Stability Analysis of the Disease Free Equilibrium}

In absence of the infectious disease, the model populations have a unique disease free steady state $E_{0}$. To find the local stability of $E_{0}$, the Jacobian of the model equations evaluated at DEF $E_{0}$ is used. Also, to determine the global stability at $E_{0}$ comparison theorem given in [16] is used. It is already shown that the DFE of model (1) - (5) is given by $E_{0}=$ $\{(\pi / \mu), 0,0,0,0\}$. Now, the stability analysis of DEF is conducted and the results are presented in the form of theorems and proofs as follows:

Theorem 1: The DFE $E_{0}$ of the system (1) - (5) is locally asymptotically stable if $\Re_{0}<1$ and unstable if $\Re_{0}>1$.

Proof: Jacobian matrix of $\left(f_{1}, f_{2}, f_{3}, f_{4}, f_{5}\right)$ with respect to $\left(S, \quad I_{u}, I_{s}, C, R\right)$ is given by

$\left.\begin{array}{cccc}-(\beta S / N) & -(\beta S / N) & 0 & \varphi \\ (\beta S / N)-a & (\beta S / N) & 0 & 0 \\ \alpha & -b & 0 & 0 \\ \epsilon & \phi & -c & 0 \\ \delta & \omega & 0 & -d\end{array}\right]$

Thus, the five eigenvalues of the matrix are determined as

$$
\begin{gathered}
\psi_{1}=-\mu \\
\psi_{2}=-d \\
\psi_{3}=-c \\
\psi_{4}=\frac{-(a+b-\beta)+\sqrt{(a+b-\beta)^{2}-4 a b\left(1-R_{0}\right)}}{2} \\
\psi_{5}=\frac{-(a+b-\beta)-\sqrt{(a+b-\beta)^{2}-4 a b\left(1-R_{0}\right)}}{2}
\end{gathered}
$$

It can be observed that the first threeeigenvalues $\psi_{1}$, $\psi_{2}$ and $\psi_{3}$ are absolutely negative quantities. However, the remaining two $\psi_{4}$ and $\psi_{5}$ are also negatives so long as the following restrictions on the parameters are valid: $a b(1-$ $\left.\Re_{0}\right)>0$ and $(a+b-\beta)^{2}>2 a b\left(1-\Re_{0}\right)$ respectively, when $\Re_{0}<1$.

Therefore, it is concluded that the DFE $E_{0}$ of the system of differential equations (1) - (5) is locally asymptotically stable if $\Re_{0}<1$ and unstable if $\Re_{0}>1$.

Theorem 2: The disease free equilibrium point $E_{0}$ of the model is globally asymptotically stable if $\mathfrak{R}_{0}<1$ and unstable if $\Re_{0}>1$.

Proof Using the comparison theorem as given in [16], the rate of change of the variables representing the disease classes of the model can be rewritten as

$$
\left[\begin{array}{l}
I_{u}{ }^{\prime} \\
I_{s}{ }^{\prime} \\
C^{0}
\end{array}\right]=(F-V)\left[\begin{array}{l}
I_{u} \\
I_{s} \\
C
\end{array}\right]-M \theta\left[\begin{array}{l}
I_{u} \\
I_{s} \\
C
\end{array}\right]
$$

Here in (10), the matrices $F$ and $V$ at the disease free equilibrium $E_{0}$ are defined as 


$$
F=\left[\begin{array}{lll}
\beta & \beta & 0 \\
0 & 0 & 0 \\
0 & 0 & 0
\end{array}\right] \text { and } V=\left[\begin{array}{ccc}
a & 0 & 0 \\
-\alpha & b & 0 \\
-\epsilon & -\phi & c
\end{array}\right]
$$

Also, and $\theta$ is non-negative matrix. However, $M=$ $\left[1-\left(S^{0} / N^{0}\right)\right]=0$ since $S^{0}=(\pi / \mu)$ and $N^{0}=(\pi / \mu)$. Therefore, the equation (10) reduces to the simplified form as

$$
\left[\begin{array}{l}
I_{u}{ }^{\prime} \\
I_{s}{ }^{\prime} \\
C^{0}
\end{array}\right] \leq(F-V)\left[\begin{array}{l}
I_{u} \\
I_{s} \\
C
\end{array}\right]
$$

Now, $(F-V)$ can be computed as

$$
F-V=\left[\begin{array}{lll}
\beta & \beta & 0 \\
0 & 0 & 0 \\
0 & 0 & 0
\end{array}\right]-\left[\begin{array}{ccc}
a & 0 & 0 \\
-\alpha & b & 0 \\
-\epsilon & -\phi & c
\end{array}\right]=\left[\begin{array}{ccc}
\beta-a & \beta & 0 \\
\alpha & -b & 0 \\
\epsilon & \phi & -c
\end{array}\right]
$$

Next, elementary row-operations are used to row-reduce the matrix in (11) to a lower triangular as in(12).

$$
F-V=\left[\begin{array}{ccc}
{[\alpha \beta+b(\beta-a)]} & 0 & 0 \\
\alpha & -b & 0 \\
\epsilon & \phi & -c
\end{array}\right]
$$

The eigenvalues of matrix (12) are found by evaluating the characteristic equationdet $[(F-V)-\psi I]=0$ as follows:

$$
\begin{gathered}
\left|\begin{array}{ccc}
{[\alpha \beta+b(\beta-a)]-\psi} & 0 & 0 \\
\alpha & -b-\psi & 0 \\
\epsilon & \phi & -c-\psi
\end{array}\right|=0 \\
{[[\alpha \beta+b(\beta-a)]-\psi](-b-\psi)(-c-\psi)=0} \\
(-b-\psi)=0,(-c-\psi)=0,[[\alpha \beta+b(\beta-a)]-\psi]=0 \\
\psi_{1}=-b, \psi_{2}=-c, \psi_{3}=[\alpha \beta+b(\beta-a)]=a b\left[\Re_{0}-1\right]
\end{gathered}
$$

The notations $\mathrm{a}, \mathrm{b}$ and $\mathrm{c}$ have been defined earlier. Here it can be observed that the first twoeigenvalues $\psi_{1}$, and $\psi_{2}$ are absolutely negative quantities. However, the remaining eigenvalue $\psi_{3}$ is also negativewhen $\mathfrak{R}_{0}<1$. Hence, the disease free equilibrium point $E_{0}$ is globally asymptotically stable if $\Re_{0}<1$ and unstable if $\Re_{0}>1$.

\subsection{The Endemic Equilibrium}

Endemic equilibrium point $E_{1}$ is a steady state solution where the disease persists in the population. For the existence and uniqueness of endemic equilibrium $E_{1}=\left\{S^{*}, \quad I_{u}^{*}, I_{s}^{*}, C^{*}, R^{*}\right\}$, its coordinates should satisfy the conditions $E_{1}=\left\{S^{*}, I_{u}^{*}, I_{s}^{*}, C^{*}, R^{*}\right\} \neq 0$, where $S^{*}>$ $0, I_{u}^{*}>0, I_{s}^{*}>0, C^{*}>0$ and $R^{*}>0$. The endemic equilibrium point is obtained by setting left hand sides of equations of the system (1) - (5) to zero. Then solved for state variables interms of the force of infection, $\lambda^{*}$ and obtain the following;

$$
\begin{aligned}
& S^{*}=(a b d \Pi) /\left[a b d\left(\lambda^{*}+\mu\right)-\lambda^{*} \varphi(b \delta+\omega \alpha)\right] \\
& I_{u}^{*}=\left(b d \Pi \lambda^{*}\right) /\left[a b d\left(\lambda^{*}+\mu\right)-\lambda^{*} \varphi(b \delta+\omega \alpha)\right] \\
& I_{s}^{*}=\left(d \alpha \Pi \lambda^{*}\right) /\left[a b d\left(\lambda^{*}+\mu\right)-\lambda^{*} \varphi(b \delta+\omega \alpha)\right]
\end{aligned}
$$

$$
\begin{gathered}
C^{*}=\left[\alpha \Pi \lambda^{*}(\epsilon b+\phi \alpha)\right] /\left[\operatorname{abcd}\left(\lambda^{*}+\mu\right)-c \lambda^{*} \varphi(b \delta+\omega \alpha)\right] \\
R^{*}=\left[\Pi \lambda^{*}(\delta b+\omega \alpha)\right] /\left[a b d\left(\lambda^{*}+\mu\right)-\lambda^{*} \varphi(b \delta+\omega \alpha)\right] \\
\text { Here } a=\epsilon+\alpha+\delta+\mu, b=\omega+\phi+\mu, c=\gamma+\mu \\
\text { and } d=\varphi+\mu .
\end{gathered}
$$

On substituting the expression for $I_{u}^{*}$ and $I_{s}^{*}$ into the force of infection, that is, $\lambda^{*}=\left[\beta\left(I_{u}^{*}+I_{u}^{*}\right)\right] / N$, characteristic polynomial of force of infection isobtainedas

$$
p\left(\lambda^{*}\right)=D_{1} \lambda^{*}+D_{2}
$$

Here $D_{1}=a b d-\varphi(b \delta+\omega \alpha)$ and $D_{2}=\mu a b d\left(1-\Re_{0}\right)$.

Clearly, $D_{1}>0$ and $D_{2} \geq 0$, when $\Re_{0}<1$ and $\lambda^{*}=$ $-D_{2} / D_{1} \leq 0$. From this, we see that, for $\mathfrak{R}_{0}<1$, there is no endemic equilibrium for this model.

Lemma 4: A unique endemic equilibrium point $E^{*}$ exists and is positive if $\Re_{0}>1$.

\section{Numerical Simulation}

In this section, the numerical simulation study of model equations (1) - (5) is carried out using the software DE Discover 2.6.4. To conduct the study, a set of meaningful values are assigned to the model parameters. These values are either taken from literature or assumed. Using the parameter values given in Table 3 and the initial conditions $S(0)=150000, I_{u}(0)=$ $50000, I_{S}(0)=30000, C(0)=12500$ and $R(0)=26250$ in the model equations (1) - (5) a simulation study is conducted and the results are given in Figures 2 and 3.

Table 3. Parameter values used in Numerical Simulations.

\begin{tabular}{lll}
\hline Parameter & Value & Reference \\
\hline$\Pi$ & 175 & assumed \\
$\mu$ & 0.1 & assumed \\
$\beta$ & 0.3 & assumed \\
$\varphi$ & 0.2 & assumed \\
$\alpha$ & 1.6 & assumed \\
$\gamma$ & 0.03 & assumed \\
$\omega$ & 0.3 & assumed \\
$\epsilon$ & 0.15 & assumed \\
$\delta$ & 0.6 & assumed \\
$\phi$ & 0.04 & assumed \\
\hline
\end{tabular}

Due to the presence of interventions, the number of susceptible cells decreases implying that, most of the susceptible cells are screened. Similarly, the number of unaware infected cells decreases. This happens because unaware cells become aware after screening. The screened infected cells initially increase and then start to diminish after the equilibrium point. This is because many people from screened class recovered through treatment. Also, the number of cells with cancer decreases and this may be due to disease induced death. Furthermore, the number of recovered cells increases because there are two ways of recovering, through immune system or treatment. With $\Re_{0}=0.5677$, implies that screening can reduce the transmission of the disease in the population when $\mathfrak{R}_{0}<1$. 


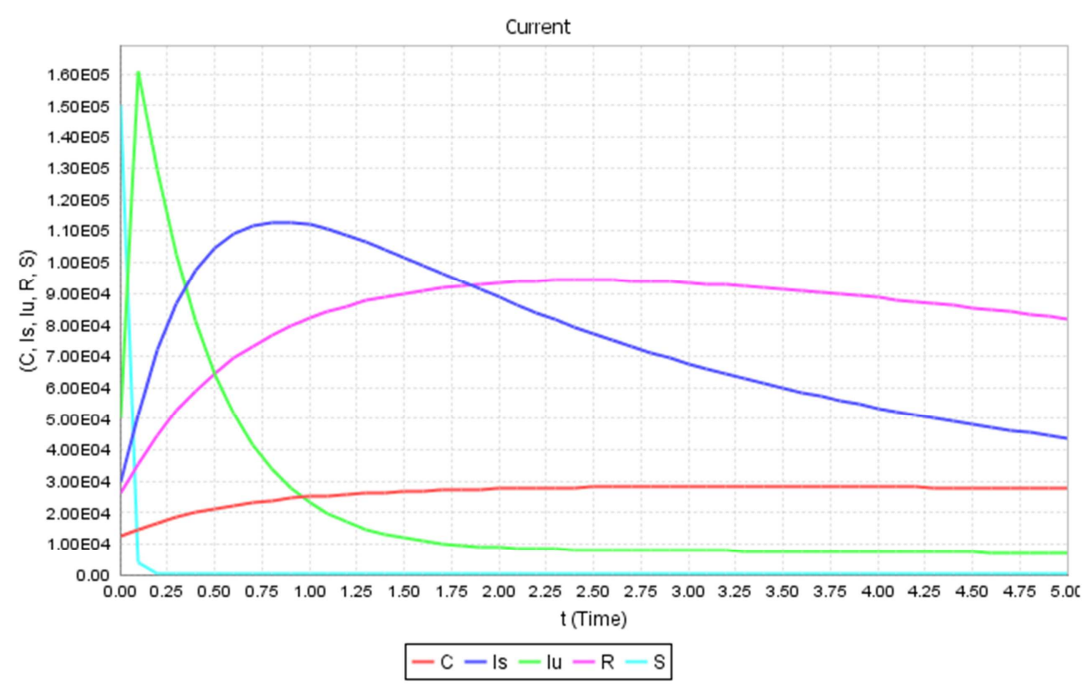

Figure 2. Simulation results grouped by parameter sets (combined of $S, I_{u}, I_{S}, C, R$ ).
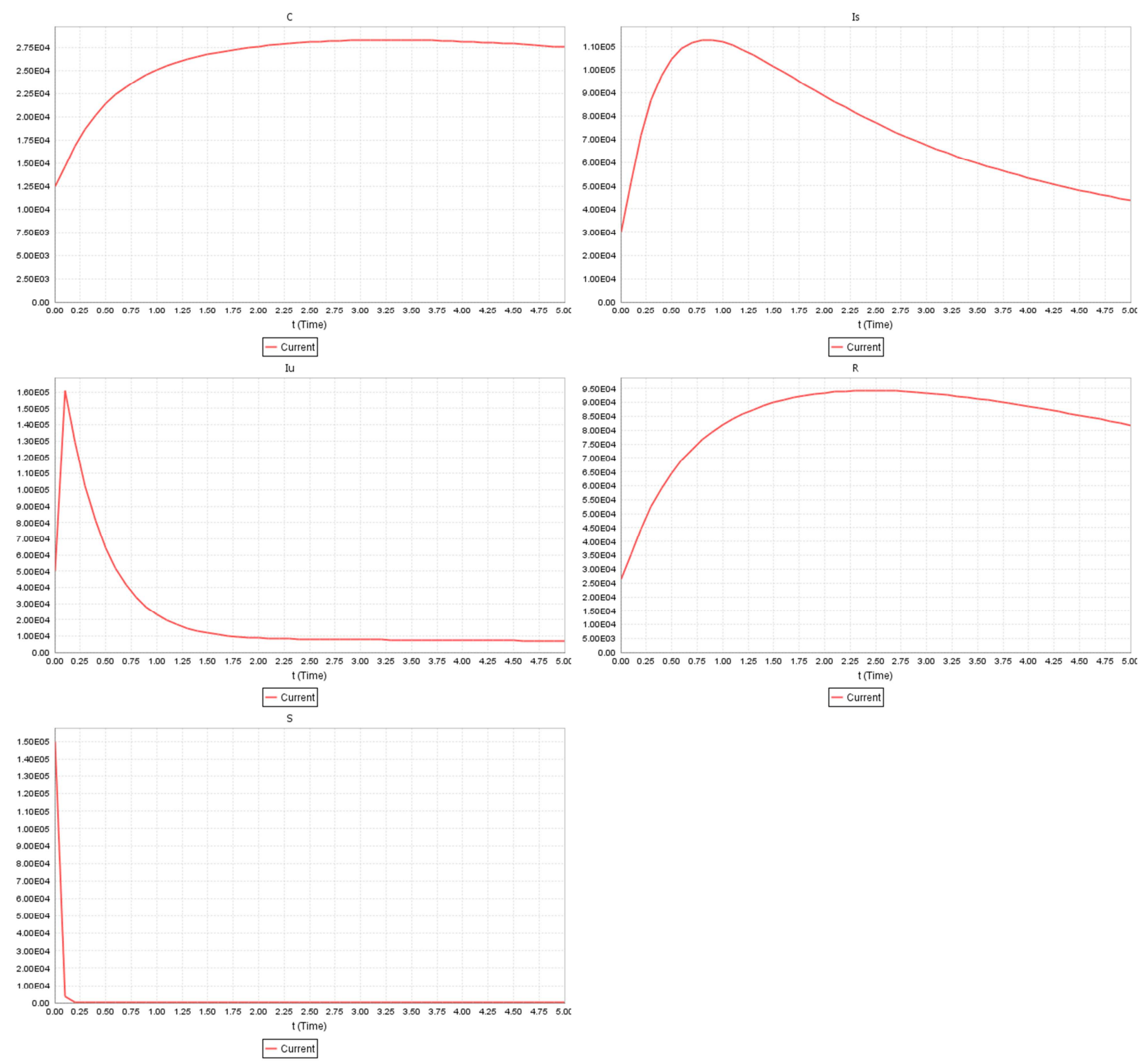

Figure 3. Simulation results grouped by variables $\left(S, I_{u}, I_{S}, C, R\right)$. 


\section{Sensitivity Analysis of Model Parameters}

We carried out sensitivity analysis in order to determine the relative significance of modelparameters on disease transmission. The analysis will enable us to find out parametersthat have high impact on the basic reproduction number and which should be targetedby intervention strategies. We perform sensitivity analysis by calculating the sensitivityindices of the basic reproduction number $\mathfrak{R}_{0}$ in order to determine whether HPV canbe spread in the population or not. These indices tell us how crucial each parameter is onthe transmission of the HPV. To investigate which parameters in the model system (1) - (5) have high impact on the $\Re_{0}$, we apply the approach presented by [15].

The explicit expression of $\Re_{0}$ is given by $\Re_{0}=$ $[\beta(b+\alpha) / a b]$. Since $\mathfrak{R}_{0}$ depends only on seven parameters, we derive an analytical expression for its sensitivity to each parameter using the normalized forward sensitivity index as by Chitnis [17] as follows:

$$
\begin{gathered}
\Upsilon_{\beta}^{R_{0}}=\left[\partial R_{0} / \beta\right] \times\left[\beta / R_{0}\right]=1 \\
\Upsilon_{\alpha}^{R_{0}}=\left[\partial R_{0} / \alpha\right] \times\left[\alpha / R_{0}\right] \\
=\alpha[a(\omega+\phi+\mu+1)-c(\epsilon+\delta+\mu+1)] /[a c] \\
\Upsilon_{\mu}^{R_{0}}=\left[\partial R_{0} / \mu\right] \times\left[\mu / R_{0}\right] \\
=\mu[a b(\omega+\phi+\alpha+1)-[a c(\omega+\phi+1)+b c(\epsilon+\delta+\alpha+1)]] /[a b c] \\
\Upsilon_{\omega}^{R_{0}}=\left[\partial R_{0} / \omega\right] \times\left[\omega / R_{0}\right] \\
=\omega[b(\phi+\alpha+\mu+1)-c(\phi+\mu+1)] /[b c] \\
\Upsilon_{\phi}^{R_{0}}=\left[\partial R_{0} / \phi\right] \times\left[\phi / R_{0}\right] \\
=\phi[b(\omega+\alpha+\mu+1)-c(\omega+\mu+1)] /[b c] \\
\Upsilon_{\epsilon}^{R_{0}}=\left[\partial R_{0} / \epsilon\right] \times\left[\epsilon / R_{0}\right]=\epsilon[(\delta+\alpha+\mu+1)] /[a] \\
\Upsilon_{\delta}^{R_{0}}=\left[\partial R_{0} / \delta\right] \times\left[\delta / R_{0}\right]=\delta[(\epsilon+\alpha+\mu+1)] /[a]
\end{gathered}
$$

Table 4. Sensitivity indices.

\begin{tabular}{ll}
\hline Parameter Symbol & Sensitivity indices \\
\hline$\beta$ & +1 \\
$\delta$ & 0.6979 \\
$\epsilon$ & 0.2020 \\
$\omega$ & -0.3743 \\
$\mu$ & -0.2972 \\
$\alpha$ & -0.0787 \\
$\phi$ & -0.0684 \\
\hline
\end{tabular}

The sensitivity indices of the basic reproductive number with respect to main parameters are arranged orderly in Table 4. Those parameters that have positive indices i.e. $\beta, \delta$ and $\epsilon$ show that they have great impact on expanding the disease in the community if their values are increasing. Due to the reason that the basic reproduction number increases as their values increase, it means that the average number ofsecondary cases of infection increases in the community. Furthermore, those parameters in which their sensitivity indices are negative i.e. $\omega, \mu, \alpha$ and $\phi$ have an influence of minimizing the burden of the disease in the community as their values increase while the others areleft constant. And also as their values increase, the basic reproduction number decreases, which leads to minimizing the endemic nature of the disease in the community.

\section{Result and Discussion}

In this paper we have studied the impact of screening as control strategies against the transmission dynamics of HPV infection. The model analysis showed that there exists a domain where the model is epidemiologically and mathematically well-posed. We have established two distinct equilibriums for the model with both local and global stability on the disease-free equilibrium. The threshold parameter that governs the disease transmission was computed using next generation matrix. Then the model was analyzed qualitatively for the existence and stability of disease free and endemic equilibrium. It was proved that the disease free equilibrium is both locally and globally asymptotically stable under certain conditions.

Numerical simulation carried out on the model revealed that, screened infected and recovered cells grows exponentially in which, after reaching equilibrium level, started dying out and became asymptotic to zero. The increase in both the screened infected and recovered cells aroused from the screening program and the treatment intervention proposed in this model on the susceptible cells and cancer cells. Also, both unaware infected cells and cancer cells died out exponentially with smooth curve overtime and became asymptotic to zero with screening program and treatment interventions in the population. Furthermore, in the absence of the HPV infection and cancer cells, the population of susceptible cells grows up to equilibrium level, achieved asymptotic stability and did not die out over time with the same proposed interventions in the model.

\section{Conclusion and Recommendation}

In this study, we formulated a mathematical model on the transmission dynamics of the HPV infection cancer by incorporating the combined effects of screening and treatment. Moreover, existence, positivity and boundedness of the formulated model are verified to illustrate that the model is biologically meaningful and mathematically well posed. In particular, the stability analyses of the model were investigated using the basic reproduction number. And also, the solution of the model equation is numerically supplementedandsensitivity analysis of the model is analyzed to determine which parameter has high impact on the transmission of diseases.

Although eradication of HPV infection remain a challenge especially in developing countries, but from results of this study we recommend that, the government should introduce education programmers on the 
importance of voluntary and routinely screening on HPV infection. Also, there is need to increase the number of hospitals to deal with HPV infection as well as cancers to ensure that, many people have access to the facilities, because HPV infection in long run results into different types of human cancers which pose serious health problem. Moreover, the future work should consider; incorporating asymptomatic and screening against HPV transmission dynamics in the model.

\section{References}

[1] R. V. Barnabas, P. Laukkanen, P. Koskela, O. Kontula, M. Lehtinen, G. P. Garnett, "Epidemiology of HPV 16 and cervical cancer in Finland and the potential impact of vaccination: mathematical modeling analyses", PLoS Med. 3 (5) (2006) e138.

[2] A. S. Bergot, A. Kassianos, I. A. Frazer, D. Mittal, "New Approaches to immunotherapy for HPV associated cancers", OPEN ACESS, Cancers (2011), 346-3495; doi $10.3390 /$ cancers 3033461 .

[3] D. R. Lowy and J. T. Schiller, "Prophylactic human Papilloma virus vaccines", $J$ C Invest (2006), 116(5): 11671173.

[4] S. L. Lee and A. M. Tameru, "A Mathematical model of Human Papilloma virus in the United States and its impact on cervical cancer", Ivyspring International Publisher J cancer (2012) 3: 262-266 doi: 10.7150/jca.4161.

[5] National Cancer Registry of South Africa. Available online: http://www.cansa.org.za/files/2015/10/NCR_Final_2010_table s1.pdf(accessed on 24 April 2018).

[6] WHO list of priority medical devices for cancer management?Geneva: World Health Organization; 2017. License: CC BY-NC-SA 3.0 IGO.

[7] Ntekim A."Cervical Cancer in Sub Sahara Africa, Topics on Cervical Cancer with an Advocacy for Prevention". Intech, 2012. Google Scholar.

[8] Jemal A, et al. "Global cancer statistics". CA Cancer JClin. 2011; 61(2): p 69-90. PubMed| Google Scholar.
[9] Addis Ababa Cancer Registry Data (2012-2014).

[10] M. Llamazares and R. J. Smith, "Evaluating human Papilloma virus vaccination pro-grams in Canada: should provincial healthcare pay for voluntary adult vaccination". BioMed Central Ltd Ottawa, Canada, (2008).

[11] L. Ribassin-Majed and R. Lounes, "A SIS Model for Human Papilloma virus transmission", 2010, hal-00555733, Version 1-14 Jan (2011).

[12] L. Ribassin-Majed, R. Lounes, S. Clemencos, "Efficacy of vaccination against HPV infections to prevent cervical cancer in France: Present assessment and Pathways to improve vaccination policies (2012)", PLoS ONE 7(3): e32251. doi: 10.-1371/journal, Pone 0032251.

[13] Kermack, W. O.; McKendrick, A. G." A contribution to the mathematical theory of epidemics". In Proceedings of the Royal Society of London A: Mathematical, Physical and Engineering Sciences, London, UK, August 1927; Volume 115 , pp. $700-721$.

[14] AkramAshyani, HajimohammadMohammadinejad, Omid RabieiMotlagh, "Stability Analysis of Mathematical Model of Virus Therapy for Cancer", Vol. 11, No. 2 (2016), pp 97-110.

[15] EshetuDadiGurmu and Purnachandra Rao Koya. Impact of Chemotherapy treatment of SITR Compartmentalization and Modeling of Human Papilloma Virus (HPV), IOSR Journal of Mathematics (IOSR - JM), Vol. 15, Issue 3, Ser. I, May - June 2019, Pp $17 \quad-\quad 29$. DOI: $10.9790 / 5728-$ $1503011729 \mathrm{http} / / /$ iosrjournals.org/iosr-jm/papers/Vol15issue3/Series-1/C1503011729.pdf

[16] TadeleTesfaTegegne, Purnachandra Rao Koya and TemesgenTibebuMekonnen, "Impact of Heterosexuality and Homosexuality on the transmission and dynamics of HIV/AIDS", IOSR Journal of Mathematics (IOSR-JM) eISSN: 2278-5728, p-ISSN: 2319-765X. Volume 12, Issue 6 Ver. V (Nov. - Dec.2016), PP 38-49.

[17] Chitnis, N., Hyman, J. M., and Cusching, J. M. (2008). Determining important Parameters in the spread of malaria through the sensitivity analysis of a mathematical Model. Bulletin of Mathematical Biology 70 (5): 1272-12. 